\title{
Segurança do processo medicamentoso em um hospital universitário: preparo e administração da equipe de enfermagem
}

\author{
Safety of the medication process in a university hospital: preparation and administration of \\ the nursing team
}

\author{
Seguridad del proceso de medicación en un hospital universitario: preparación y \\ administración del equipo de enfermería
}

Vanessa Da Conceição Santos ${ }^{2}$, Cyntia Maria Santos ${ }^{2}$, Juliana de Oliveira Musse Silva ${ }^{3}$, Sílvia Maria da Silva Sant'ana Rodrigues ${ }^{2}$, Max Cruz da Silva ${ }^{4}$, Ruth Cristini Torres ${ }^{6}$, Marcel Vinícius Cunha Azevedo ${ }^{5}$, Ângela Maria Melo Sá Barros ${ }^{7}$, Ana Fátima Souza Melo de Andrade ${ }^{8}$, Weber de Santana Teles ${ }^{1 *}$.

\section{RESUMO}

Objetivo: Conhecer o processo de trabalho das equipes de saúde durante a administração de medicamentos em um Hospital Universitário. Métodos: Trata-se de uma pesquisa observacional, descritiva e com abordagem quantitativa. A pesquisa ocorreu em dois momentos distintos. No primeiro momento, foi aplicado um questionário para 55 técnicos de enfermagem. O segundo momento da pesquisa caracterizou-se como observacional, no qual as pesquisadoras realizaram o acompanhamento de 28 técnicos de enfermagem, escolhidos aleatoriamente, durante a execução das atividades relacionadas ao preparo, checagem, administração de medicamentos pelos colaboradores. Resultados: Esse estudo possibilitou identificar as principais fragilidades durante o processo de medicação de um hospital universitário. O ruído nos setores emergiu como um dos principais fatores ambientais da instituição que interferem na etapa de preparação, bem como inadequação na lavagem das mãos e a interrupção por conversas paralelas. Conclusão: Sob outro aspecto, durante a administração dos medicamentos observou-se que algumas ações importantes para a segurança do paciente não são realizadas por alguns profissionais, tais como as relacionadas à identificação do paciente conferem o nome do paciente na pulseira de identificação não atendendo, monitoramento do paciente e descarte de materiais.

Palavras-chave: Acesso a medicamentos essenciais, Tecnologias em saúde, Adesão à medicação, Assistência centrada no paciente.

\begin{abstract}
Objective: To know the work process of health teams during medication administration in a University Hospital. Methods: This is an observational, descriptive research with a quantitative approach. The research took place at two different times. At first, a questionnaire was applied to 55 nursing technicians. The second moment of the research was characterized as observational, in which the researchers monitored 28 nursing technicians, randomly chosen, during the execution of activities related to the preparation, checking, and administration of medication by the collaborators. Results: This study made it possible to identify the main weaknesses during the medication process at a university hospital. The noise in the sectors emerged as one of the main environmental factors of the institution that interfere in the preparation stage, as well as inadequacy in hand washing and interruption by parallel conversations. Conclusion: In another aspect, during the administration of the drugs, it was observed that some important actions for the safety of the patient are not carried out by some professionals, such as those related to patient identification, giving the patient's name on the identification bracelet, not attending, patient monitoring and material disposal.
\end{abstract}

Keywords: Access to essential medicines, Health technologies, Medication adherence, Patient-centered care.

\section{RESUMEN}

Objetivo: Conocer el proceso de trabajo de los equipos de salud durante la administración de medicamentos en un Hospital Universitario. Métodos: Se trata de una investigación observacional, descriptiva, con enfoque cuantitativo. La investigación se llevó en dos momentos diferentes. Inicialmente, se aplicó un cuestionario a 55 técnicos de enfermería. El segundo momento de la investigación se caracterizó como observacional, en el que los investigadores acompañaron a 28 técnicos de enfermería, elegidos al azar, durante la ejecución de actividades relacionadas con la preparación, control y administración de medicamentos por parte de los colaboradores. Resultados: Este estudio permitió identificar las principales debilidades durante el proceso de medicación en un hospital universitario. El ruido en los sectores emergió como uno de los principales factores ambientales de la institución que interfieren en la etapa de preparación, así como la

\footnotetext{
${ }^{1}$ Centro de Hemoterapia de Sergipe (HEMOSE), Aracaju - SE. *E-mail: arteecura@hotmail.com

2 Centro Universitário Uninassau, Aracaju - SE.

${ }^{3}$ Hospital Universitário UFS Ebserh, Aracaju - SE.

${ }^{4}$ Faculdade Pio Décimo de Canindé (FAPIDE), Canindé de São Francisco - SE.

${ }^{5}$ Universidade Tiradentes (UNIT), Aracaju - SE.

${ }^{6}$ Instituto de Hematologia e Hemoterapia de Sergipe (IHHS), Aracaju - SE.

${ }^{7}$ Universidade Federal do Rio de Janeiro, Rio de Janeiro - RJ

${ }^{8}$ Centro Universitário Estácio de Sergipe, Aracaju - SE.
} 
inadecuación en el lavado de manos y la interrupción por conversaciones paralelas. Conclusión: En otro aspecto, durante la administración de los medicamentos, se observó que algunas acciones importantes para la seguridad del paciente no son realizadas por algunos profesionales, como las relacionadas con la identificación del paciente, consignando el nombre del paciente en el brazalete de identificación no asistencia, seguimiento de pacientes y disposición de material.

Palabras clave: Acceso a medicamentos esenciales, Tecnologías sanitarias, Cumplimiento de la medicación, Atención dirigida al paciente.

\section{INTRODUÇÃO}

A gestão dos processos de medicação nos estabelecimentos de saúde é de grande complexidade, uma vez que o mesmo é composto por muitas etapas (organização e gerenciamento, seleção, aquisição, estoque, dispensação, administração e monitoramento) (FORTE ECN, et al., 2017). As etapas realizadas para gerir medicações são realizadas por diferentes profissionais da área administrativa e da saúde, o que torna necessário um monitoramento contínuo das ações relacionadas aos procedimentos para a prevenção de possíveis desfechos negativos decorrentes de erros humanos (MINISTÉRIO DA SAÚDE, 2019)

Os Erros de Medicação (EM), frequentemente acontecem nos serviços de saúde, principalmente em ambiente hospitalar, e são caracterizados como eventos adversos potencialmente evitáveis, os quais possuem a capacidade de causar agravos à saúde do paciente (transitórios, permanentes ou morte) (SOUZA MJ, et al., 2017). Além de afetar a segurança do paciente, geram danos para os estabelecimentos de saúde, e elevação dos custos em decorrência do aumento do tempo de internação hospitalar (REIS UOP, et al., 2020).

Um evento que envolve a seguridade do indivíduo que está sendo atendido, no estabelecimento assistencial de saúde, é conceituado como uma ocorrência ou conjuntura que pode ocasionar, ou ocasionou prejuízo para a saúde do paciente, podendo acontecer, através de comportamentos mecânicos ou intencionais. Tal eventualidade está sempre correlacionada a um séquito de causas que contribuem de maneira decisiva para segurança do paciente, estando relacionado a eventos externos ao método de atenção, o surgir a partir da insipiência dos profissionais de saúde, através do indivíduo que está sendo atendido, ambiente de trabalho subdimensionado, climatização, ou não conformidades dos procedimentos operacionais padrão (WORLD HEALTH ORGANIZATION (WHO), 2009).

Os prejuízos pertinentes ao atendimento em saúde, pode ocasionar ao paciente e a sua parentela decorrências no âmbito físico, social e da psique. Para a corporação acarreta uma enorme repercussão, gerado pela minimização de eficiência de pessoas, assim como aumento da demanda nos serviços de atendimentos de saúde, expressados por alargamento de internamentos, recondução ao hospital, carência de exames para perquirição clínica e fisiológica, terapêutica medicamentosa entre outros gastos. Entretanto o retorno do paciente ao sistema de atendimento abala a confiança dos protocolos de atenção dos serviços básicos de saúde, assim como as instituições que oferecem o serviço (SLAWOMIRSKI L, et al., 2017).

Atendendo os enredamentos e a variação dos atendimentos ofertados, os erros de procedimentos nos atendimentos a saúde, na maioria das vezes estão correlacionados a situações de organização, sapiência, que contabilizada à possibilidade de falha, pode ocasionar em erros dispensáveis aos indivíduos que estão fazendo uso dos serviços de saúde (ZSIFKOVITS J, et al., 2016).

A equipe multidisciplinar de enfermagem compõe como um óbice significativo para precaução de inexatidões, já que são ajuizados pela manipulação e administração dos fármacos, assim como o monitoramento de possíveis efeitos adversos decorrentes do tratamento podendo a todo instante estar envolvida em situações de risco (VÓRIA JO, et al., 2020).

No que diz respeito a terapêutica medicamentosa, devido ao seu hermetismo dos procedimentos operacionais que o constitui, reclama a colaboração do grupo multiprofissional que campeiam nos serviços de saúde que conta com a prescrição do médico, seguidamente a análise e revisão do farmacêutico, que sem demora seleciona os fármacos e entrega aos auxiliares de farmácia, em seguida aos técnicos da área de enfermagem que irão manipular, realizar a administração e monitorização da fármacos após administrados. O envolvimento de todos que atuam nessa complexidade é de suma importância afim de que a segurança do paciente não seja ameaçada (SIMAN AG, et al., 2019). 
Nesse sentido, alguns estudos na literatura demonstram a importância da supervisão dessas práticas visando à segurança do paciente. Dessa forma, vêm identificando os erros mais frequentes cometidos pelas equipes durante o processo de terapia medicamentosa, visando conhecer os principais fatores de risco e impactos negativos na saúde dos pacientes, e sobretudo subsidiar novas estratégias de prevenção e mitigação dos danos (SILVA ACA, et al., 2016).

Um estudo desenvolvido Pires AOM, et al. (2017) com 162 profissionais de enfermagem no Maranhão, identificou como os principais fatores causais de EM: a falta de atenção do profissional, conhecimento e prescrição ilegíveis. Aquino FFR, et al. (2015) ressaltam que a qualidade das prescrições médicas também pode contribuir para os EM à omissão de informações ou escrita que possibilita interpretação dúbia, dão margem para a administração incorreta de dosagem, via e escolha de medicação inadequada.

A manipulação e administração inadequada de medicamentos podem acontecer em decorrência de fatores extrínsecos e intrínsecos aos profissionais envolvidos durante o processo (MINISTÉRIO DA SAÚDE, 2013). Os extrínsecos são aspectos inerentes à aquisição de materiais, bem como àqueles referentes ao gerenciamento da assistência de enfermagem, como déficit de recursos humanos que resulta em sobrecarga de trabalho e favorecem a ocorrência de iatrogenias (COSTA CO, et al., 2021).

Sob outra perspectiva, os fatores intrínsecos estão relacionados ao conhecimento do profissional quanto ao acondicionamento, preparo e administração do medicamento, e, sua iniciativa em buscar a educação continuada e/ou permanente para subsidiar suas práticas (MAGALHÃES AMM, et al., 2015). Dentro das inúmeras atribuições relativo a cautela, inexatidões pertinentes ao fármaco, indicam para a falta de políticas de saúde e da indústria farmacêutica direcionadas ao suporte das especificidades das comunidades atendida (BICA TFS, et al., 2017).

Neste contexto, a pesquisa teve como objetivo conhecer o processo de trabalho das equipes de saúde durante a administração de medicamentos em um Hospital Universitário. Para tal buscou-se analisar o processo de preparo e administração de medicamentos pela equipe de enfermagem; identificar os principais fatores que influenciam a ocorrência de erros na administração de medicamentos nos setores e realizar o levantamento das necessidades de capacitações referidas pela equipe de enfermagem para uma prática de administração medicamentosa mais segura.

\section{MÉTODOS}

Trata-se de uma pesquisa observacional, descritiva e com abordagem quantitativa. A pesquisa ocorreu no segundo semestre ano de 2020 e em dois momentos distintos. No primeiro momento, foi aplicado um questionário, elaborado e disponibilizado por meio do Google Forms, para técnicos de enfermagem dos setores de Clínica Médica 1, Clínica Médica 2, Clínica Cirúrgica 1 e Unidade de Terapia Intensiva (UTI) dos turnos da manhã, tarde e noite. Participaram do estudo 55 técnicos de enfermagem, do total de 91 . Foram excluídos os profissionais que não responderam todas as perguntas presentes no instrumento de coleta de dados desenvolvidos pelas pesquisadoras, que estivam de licença ou férias no momento da coleta dos dados, ou não autorizaram o uso de respostas.

O questionário continha perguntas fechadas relacionadas a identificação do participante, turno e setor de trabalho, tipo de vínculo empregatício, realização ou não de cursos sobre atualização de administração de medicamentos, o conhecimento do profissional relacionado aos Procedimentos Operacionais Padrões (POPs) sobre administração de medicações, fatores ambientais relacionados a iluminação, ruídos e organização do setor, modo de preparo das medicações, monitoramento dos sinais vitais dos pacientes antes a após a administração das medicações, registro no prontuário, uso de adornos e celular durante a jornada de trabalho.

O segundo momento da pesquisa caracterizou-se como observacional, no qual as pesquisadoras realizaram o acompanhamento de 28 técnicos de enfermagem, escolhidos aleatoriamente, durante a execução das atividades relacionadas ao preparo, checagem, administração de medicamentos pelos colaboradores.

Para essa fase do estudo, foi elaborado um roteiro de observação contendo campos relacionados a identificação de fatores como: acondicionamento dos medicamentos, ambientais (organização, iluminação e 
ventilação do local que está sendo preparado o medicamento); preparo dos medicamentos (checagem, medidas adotadas para segurança do paciente, técnica de manipulação e identificação do medicamento); administração de medicamentos (adoção de medidas para segurança do paciente, técnica de administração e monitoramento de efeitos adversos e cumprimento dos horários estabelecidos no aprazamento) e registro (anotações de enfermagem quanto à administração e intercorrências durante os procedimentos); conhecimento dos profissionais sobre o medicamento e a prescrição de medicamentos (prescrição incompleta ou ilegível, ou redação contendo erros).

Os resultados foram tabulados e organizados em tabela confeccionada no Excel e analisados por meio do Programa Bioestat versão 5.0, utilizando-se estatística descritiva.

A pesquisa respeitou os preceitos éticos estipulados pela resolução 466/2012 que dispõe sobre as diretrizes e normas regulamentadoras de pesquisas envolvendo seres humanos. As ações do projeto foram aprovadas pelo Comitê de Ética da Universidade Federal de Sergipe, e, com registro na plataforma Brasil sob Parecer Consubstanciado de № 3.210.228 e CAAE 06195418.0.0000.5546.

\section{RESULTADOS E DISCUSSÃO}

Participaram da primeira etapa da pesquisa o total de 55 técnicos de enfermagem, destes, $80 \%$ eram do sexo feminino e $20 \%$ do sexo masculino. As perguntas do questionário foram distribuídas em três blocos principais: aspectos ambientais, de preparo e administração de medicamentos e os pessoais (Tabela 1).

Tabela 1 - Preparo e administração dos medicamentos pelos Técnicos de Enfermagem.

\begin{tabular}{|c|c|c|}
\hline Aspectos ambientais & Adequado (\%) & Inadequado (\%) \\
\hline lluminação & $(74,5 \%)$ & $(25,5 \%)$ \\
\hline Ruído & $(21,8 \%)$ & $(36,4 \%)$ \\
\hline Climatização & $(72,7 \%)$ & $(27,2 \%)$ \\
\hline Limpeza & $(80 \%)$ & $(20 \%)$ \\
\hline Organização & $(78,2 \%)$ & $(21,8 \%)$ \\
\hline Local para higienização das mãos & $(94,5 \%)$ & $(5,5 \%)$ \\
\hline Aspectos do Preparo e Administração & Adequado (\%) & Inadequado (\%) \\
\hline Identificação do paciente & $(96,4 \%)$ & $(3,6 \%)$ \\
\hline Interrompe o preparo & $(16,4 \%)$ & $(83,6 \%)$ \\
\hline Organização das medicações individuais & $(80 \%)$ & $(20 \%)$ \\
\hline Identifica os medicamentos com etiquetas & $(100 \%)$ & $(0)$ \\
\hline Realiza a dupla checagem & $(98,2 \%)$ & $(1,8 \%)$ \\
\hline Realiza o 11 certos & $(89,1 \%)$ & $(10,9 \%)$ \\
\hline Realiza documentação e registro & $(100 \%)$ & $(0)$ \\
\hline Monitora sinais clínicos e sinais vitais & $(50,9 \%)$ & $(49,1 \%)$ \\
\hline Confere horários aprazados pelo enfermeiro & $(96,4 \%)$ & $(3,6 \%)$ \\
\hline Realiza o descarte correto & $(100 \%)$ & (0) \\
\hline Aspectos Pessoais & SIM (\%) & NÃO (\%) \\
\hline Conhecimento sobre Pop's sobre administração de medicamentos & $(87,3 \%)$ & $(12,7 \%)$ \\
\hline Participação em atualização sobre administração de medicamentos & $(69,1 \%)$ & $(30,9 \%)$ \\
\hline Possui outro vínculo empregatício & $(70,9 \%)$ & $(29,1 \%)$ \\
\hline Instituição permite uso de celular & $(81,8 \%)$ & $(18,2 \%)$ \\
\hline Uso de adornos no turno de trabalho & $(14,5 \%)$ & $(85,5 \%)$ \\
\hline
\end{tabular}

Fonte: Santos VC, et al., 2022.

Desses profissionais, $70,9 \%$ possuem outro vínculo empregatício, $87,7 \%$ informaram conhecimento sobre o POP de administração de medicações da instituição, 69,1 \% participaram de cursos de atualização para a práticas seguras na administração de medicações. Vale ressaltar que $81,8 \%$ dos técnicos relataram que a instituição permite o uso de celular durante a jornada laboral e $84,5 \%$ não faz uso de adornos durante a jornada de trabalho. 
De acordo Pereira CDFD, et al. (2016) existem vários fatores que podem levar um profissional ao erro na hora do preparo e administração de medicamentos. A grande maioria das instituições de saúde possuem ritmo acelerado de trabalho e pouco tempo para descanso, fatos estes que podem reduzir a atenção, por outro lado, baixa remuneração que obrigam os profissionais a terem mais de um vínculo empregatício em turnos seguidos de trabalho para aumentar a sua renda mensal (GOMES ATL, et al., 2016).

Pesquisa efetuada por Figueiredo ML, et al. (2018), demonstrou que inúmeros são os equívocos que ocorrem a terapêutica medicamentosa, e que os mesmos estão relacionados a imprecisões de hora de administração, procedimentos técnicos, infusão do fármaco, fórmula do fármaco, concentração, diluição, ordenação. A associação entre sobrecarga de trabalho e a falta de atenção, somada muitas vezes a inexperiência, prescrição dos fármacos que é imprescindível que esta seja legível, potencializam a ocorrência de erros (MATIAS EO, et al. 2015).

Quando questionados sobre a interferência dos fatores ambientais durante o processo de preparo das medicações, observou-se que a maior parte dos participantes referiu adequação quanto a acessibilidade ao local e materiais necessários para higienização das mãos $(94,5 \%)$, seguido da limpeza do local de preparo das medicações $(80 \%)$ e organização do setor $(78,2 \%)$. Os aspectos ambientais conferidos como mais inadequados foram o ruído $(36,4 \%)$ e a climatização $(27,2 \%)$.

No que concerne aos procedimentos realizados durante o preparo e administração dos medicamentos, todos os participantes (100\%) referiram que identificam os medicamentos com etiqueta, documentam e registram a administração das medicações e realizam o descarte correto dos materiais. Um estudo realizado por Tomasi YT, et al. (2015) identificou que as inadequações que mais cometem durante este processo estão a interrupção durante o preparo $(83,6 \%)$ e a falta de monitoramento do paciente quanto aos sinais clínicos de eventos adversos (49,1\%).

A segunda etapa constituiu na observação da rotina de 28 profissionais, as observações foram divididas em dois blocos principais: durante o preparo (fatores ambientais e características pessoais) e administração (características pessoais) (Tabela 2).

Quadro 2 - Preparo e administração dos medicamentos pelos Técnicos de Enfermagem.

\begin{tabular}{|c|c|c|}
\hline \multicolumn{3}{|c|}{ PREPARO } \\
\hline Fatores ambientais & Adequado (\%) & Inadequado (\%) \\
\hline Iluminação & $(100 \%)$ & $(0)$ \\
\hline Ruído & $(35,7 \%)$ & $(64,3 \%)$ \\
\hline Climatização & $(92,9 \%)$ & $(7,1 \%)$ \\
\hline Espaço & $(35,7 \%)$ & $(64,3 \%)$ \\
\hline Limpeza & $(92,9 \%)$ & $(7,1 \%)$ \\
\hline Organização & $(57,1 \%)$ & $(42,9 \%)$ \\
\hline Local para higienização das mãos & $(32,1 \%)$ & $(67,9 \%)$ \\
\hline Características Pessoais & Adequado (\%) & Inadequado (\%) \\
\hline Identificação do paciente & $(60,7 \%)$ & $(39,3 \%)$ \\
\hline Higienização das mãos & $(32,1 \%)$ & $(67,9 \%)$ \\
\hline Organização do material & $(39,3 \%)$ & $(60,7 \%)$ \\
\hline Preparo do medicamento & $(46,4 \%)$ & $(56,3 \%)$ \\
\hline \multicolumn{3}{|c|}{ ADMINISTRAÇÃO } \\
\hline Características Pessoais & Adequado (\%) & Inadequado (\%) \\
\hline Segurança do paciente & $(10,7 \%)$ & $(89,3 \%)$ \\
\hline Higienização das mãos & $(32,1 \%)$ & $(67,9 \%)$ \\
\hline Procedimento durante a aplicação & $(17,9 \%)$ & $(82,1 \%)$ \\
\hline Descarte inadequado do material após procedimento & $(53,6 \%)$ & $(46,4 \%)$ \\
\hline Documentação e Registro & $(100 \%)$ & (0) \\
\hline Monitora o paciente & $(14,3 \%)$ & $(85,7 \%)$ \\
\hline
\end{tabular}

Fonte: Santos VC, et al., 2022. 
Durante o preparo de medicações, quanto aos fatores ambientais observou-se maior adequação da iluminação (100\%), seguido da climatização $(92,9 \%)$ e limpeza $(92,9 \%)$. Sob outro aspecto, os fatores ambientais considerados como mais inadequados foram ruído (64,3\%) e o espaço utilizado (64,3\%). Referente à categoria de características pessoais observou-se melhor adequação a identificação do paciente $(60,7 \%)$ e de inadequação a não higienização das mãos conforme técnica preconizada pela Anvisa (67,9\%), seguido da interrupção do preparo do medicamento com conversas ou pausas $(60,7 \%)$.

O estudo desenvolvido Sassaki RL, et al. (2019) cujo objetivo era observar 121 rodadas de medicação (preparo, administração e documentação) realizadas por 15 enfermeiros e nove técnicos de enfermagem em Unidade de Terapia Intensiva Neonatal no interior de São Paulo, demonstrou o total de 127 interrupções, principalmente na fase de preparo $(97 / 76,4 \%)$. A principal fonte da interrupção foi pela equipe de enfermagem (48/37,8\%). Já as principais causas: troca de informações (54/42,5\%), conversa paralela (28/22\%).

$\mathrm{Na}$ administração de medicamentos no quesito adequação observou-se que $100 \%$ dos profissionais realizam adequadamente a documentação e registro, o que também se assemelha a pesquisa realizada por Telles F, et al. (2004), porém a maioria não confere o nome do paciente na pulseira de identificação $(89,3 \%)$, não atendendo dessa forma as diretrizes de segurança preconizadas pela Anvisa.

Uma pesquisa realizada por Mendes JR, et al. (2018) realizada em um serviço de emergência de um hospital universitário da cidade de São Paulo (SP), também constituída por observações, um total de 303, tratando sobre os preparos de medicação endovenosa realizado por profissionais de enfermagem, durante a etapa da organização de materiais foram observados erros como a falta de higienização das mãos $(70,29 \%)$ e a não utilização de técnicas assépticas (80,85\%). No momento da administração da medicação, $81,18 \%$ dos profissionais não realizaram a higienização das mãos, e $84,81 \%$ não utilizaram qualquer tipo de técnica asséptica.

Os resultados também corroboram com o estudo de Oliveira JKA, et al. (2018) cuja pesquisa realizada em uma Unidade de Terapia Intensiva por meio de 3402 observações também observou inconsistências nas ações envolvendo a higienização das mãos durante a administração de medicamentos não alcançando taxas de conformidades específicas menores que 70\%.

Estudos realizados na Europa ocidental, na monarquia constitucional parlamentar democrática da Holanda, foi observado em prontuários de pacientes internados, que cerca de 15,2\% dos episódios antagônico apontados tem relação aos fármacos, sendo que 18,2\% foram julgados como refugáveis. Contudo efetuando uma varredura das ocorrências previamente, no decurso e posteriormente a internação na ambiência de saúde, observou-se que $35,1 \%$ das inexatidões sucederam no espaço de tempo em que o paciente se encontrava internado, sendo que 47,9\% foram críticos, $50 \%$ julgados refugáveis (CROFT LD, et al.,2017).

Outras perquirições envolvendo países de regiões diferentes, observou os episódios de ambiguidade, demonstrando que as maiores porcentagens foram de inexatidões, seguido de administração dos fármacos (SCHWENDIMANN R, et al., 2018). Averiguações realizada um país nórdico situado na região da FinoEscandinávia, no norte da Europa, demonstrou que a maioria das imprecisões foram referentes a administração medicamentosa (HÄRKÄNEN M, et al., 2015). Investigação realizada com profissionais da área de saúde, na República Islâmica no Golfo Pérsico (Árabe), observou que o maior índice de incorreções foi devido a quantidade de desacertada de fármaco administrado, seguido de receita prescrita incorretamente.

Apuração de estudos, enfatiza o quantitativo de indivíduos atendidos, proporções de fármacos escritos, categorização, identificação, manipulação, solvência, distribuição, complexidades e contratempos de intercomunicação na equipe multidisciplinar são apontados como criticidade. Ao se abordar acerca dos procedimentos operacionais padrão referente aos serviços supracitados os profissionais informaram que incontáveis fatores contribuem para que suceda as inexatidões, sendo que a sobrecarga de serviço, entre outros afazeres, executados no período do atendimento, assim como o quantitativo de atendimentos tem contribuído de maneira decisiva para a constância dos serviços prestados (TEIXEIRA TCA, et al., 2010).

Capacitação, ensaios, são de altamente significativos e devem ser efetuados constantemente envolvendo toda a equipe profissional que atuam indiretamente e diretamente nessa ambiência, tendo em vista a promoção da seguridade do paciente e a melhoria do sistema da qualidade dos serviços prestados. Tais 
ensaios e práticas são implantados e implementados visando conter os prováveis riscos, auxiliando desmesuradamente na resolutividade das adversidades que se acham em desacordo com a expectação da cooperação do sistema de saúde com segurança (WHO, 2009).

A utilização de recentes técnicas com abordagem cientificas, envolvendo equipamentos, podem auxiliar para ampliar o acerto e minimizar os erros na terapêutica dos fármacos, entretanto julga-se que essas metodologias devem ser monitoradas e avaliadas constantemente, afim de que possam produzir indicadores que contribuam para redução do quantitativo de serviços efetuados pelo profissional de saúde, ou delineamento de assessoramento aos pacientes atendidos, tendo em vista a ausência do profissional no monitoramento ao indivíduo (MAGALHÃES AMM et al., 2015).

O propósito da análise sistemática do sistema do sistema de gestão da qualidade, é a determinação permanente dos serviços prestados pela equipe multiprofissional de saúde, observando celeremente as inexatidões, retificando previamente. O acompanhamento das não conformidades encontradas nos serviços de saúde, obtém-se através de um séquito de indicadores de qualidades e são instrumentos portentosos nos procedimentos de melhoria constantes dos serviços prestados (MENDES W e MOURA MLO, et al., 2017).

A estrutura dos procedimentos de organização de segurança requer que seja elencado justificações e resultâncias que tenham correlações a modelos aceitos fundamentados em normas e regulamentação vigentes (WATSON G, 2016). Entretanto os imprevistos e inconvenientes podem ser evitados seguindo as normas e regulamentos, que se ajustam a partir de comportamentos e atitudes que vai desde as estruturas organizacionais, limpezas e desinfecções das mãos e utensílios e equipamentos utilizados nos atendimentos de saúde, e recognição legitima do indivíduo que está sendo atendido (SILVA AT, et al., 2018).

De acordo com as medidas de ações efetuadas para impedir as inexatidões medicamentosas, sobressai os procedimentos operacionais de seguridade na manipulação e preparação do fármaco, uso da receita médica eletronicamente, participação ativa do profissional de farmácia e qualificação continuada. Não obstante a equipe de enfermagem em sua múltipla atuação nos estabelecimentos assistencial de saúde exerce uma significativa participação nos procedimentos medicamentosos, em especial na etapa de finalização, que resulta na administração do fármaco aos indivíduos que estão sendo atendidos, e esta etapa supracitada vai desde a manipulação a infusão, seguido do acompanhamento da resposta do fármaco no paciente (FIGUEIREDO ML, et al., 2018).

A agência experiente em saúde, e auxiliar à Organização das Nações Unidas, vem sensibilizando os elementos interligados aos atendimentos de saúde, com o foco no indivíduo que está sendo atendido, como significativa tática para propiciar prerrogativas relevantes para os estabelecimentos assistenciais de saúde e para os indivíduos que fazem uso dos serviços prestados, estimulando, e instigando alterações na maneira com os sistemas de saúde são assegurados, administrados e oferecidos (WHO, 2015).

\section{CONCLUSÃO}

Esse estudo possibilitou identificar as principais fragilidades durante o processo de trabalhos das equipes de saúde de um Hospital Universitário. Foi observado em algumas ações o não atendimento ao protocolo de segurança, além de reclamações por parte do profissional acerca adequação quanto a acessibilidade ao local e materiais necessários para higienização das mãos. Considera-se que as observações supracitadas possam auxiliar os diretores, administradores e profissionais de saúde, acerca da seguridade dos pacientes atendidos nos estabelecimentos assistências de saúde, assim como sensibilizar nas tomadas de decisões, fomentando métodos que repliquem as demandas dos estabelecimentos que administram.

\section{REFERÊNCIAS}

1. AQUINO FFR, et al. Incidentes relacionados a medicamentos em uma instituição hospitalar: subsídios para a melhoria da gestão. Revista Enfermagem UERJ, 2015; 23(5): 616-621.

2. BICA TFS, et al. Characteristics of patient safety incidents notified in a pediatric intensive care unit. Rev Enferm UFPE Online, 2017; 11(10): S4206-16.

3. COSTA CO, et al. Segurança do paciente pediátrico no processo de administração de medicamento endovenoso. Revista Enfermagem em Foco, 2020; 11(4): 194-201. 
4. CROFT LD, et al. Frequency of Adverse Events Before, During, and After Hospital Admission. South Med J, 2016; 109(10): 631-635.

5. FIGUEIREDO ML, et al. Análise da ocorrência de incidentes notificados em hospital-geral. Rev Bras Enferm, 2018; 71(1): 121-30.

6. FORTE ECN, et al. A Relação Da Enfermagem Com Os Erros De Medicação: Uma Revisão Integrativa. Cogitare Enfermagem, 2017; 21(5): 1-10.

7. GOMES ATL, et al. Erros Na Administração De Medicamentos: Evidências E Implicações Na Segurança Do Paciente. Cogitare Enfermagem, 2016; 21(3): 1-11.

8. HÄRKÄNEN M, et al. The factors associated with medication errors in adult medical and surgical inpatients: a direct observation approach with medication record reviews. Scand J Caring Sci, 2015; 29(2): 297-306.

9. MATIAS EO. Avaliação da pratica de enfermagem no processo de administração de medicamento intravenoso na pediatria. Dissertação (Pós-graduação em enfermagem). Universidade Federal do Ceara, Fortaleza, 2015: 50-59.

10. MAGALHÃES AMM, et al. Processos de medicação, carga de trabalho e a segurança do paciente em unidades de internação. Revista da Escola de Enfermagem da USP, 2015; 1(49): 43-50.

11. MENDES JR, et al. Tipos e frequência de erros no preparo e na administração de medicamentos endovenosos. Einstein (São Paulo), 2018; 16(3): eAO4146.

12. MENDES W, MOURA MLO. Estratégias para a segurança do paciente em serviços de saúde. In: Ministério da Saúde, Agência Nacional de Vigilância Sanitária. Assistência Segura: Uma Reflexão Teórica Aplicada à Prática. Brasília: Anvisa, 2017: 47-63.

13. MINISTÉRIO DA SAÚDE. Agência Nacional de Vigilância Sanitária (ANVISA). Protocolo De Segurança Na Prescrição, Uso E Administração De Medicamentos. 2013. Disponível em: https://www.hospitalsantalucinda.com.br/downloads/prot_meficamentos.pdf. Acessado em: Agosto de 2020.

14. MINISTÉRIO DA SAÚDE. Boletim de Farmacovigilância no. 8. Ano 2019. 2019. Disponível em: https://www.gov.br/anvisa/pt-br/centraisdeconteudo/publicacoes/fiscalizacao-emonitoramento/farmacovigilancia/boletins-de-farmacovigilancia/boletim-de-farmacovigilancia-no-08.pdf/view. Acessado em: Agosto de 2020. Acessado em: 21 de março de 2021

15. OLIVEIRA JKA, et al. Patientsafety in nursing care during medication administration. Rev. Latino-Am. Enfermagem, 2018; 26(4): e3017.

16. PEREIRA CDFD, et al. Segurança Do Paciente: Avaliação Do Sistema De Medicação Por Enfermeiros Utilizando Análise Fotográfica. Enfermagem em Foco, 2017; 7(1): 76-80.

17. PIRES AOM, et al. Elaboration and Validation of the Medication Prescription Safety Checklist. Revista Latinoamericana de Enfermagem, 2017; 1(25): 1-9.

18. REIS UOP, et al. Erros no preparo e na administração de medicamentos intravenosos. Rev baiana enferm, 2020; 34(2): e3645.

19. SASSAKI RL, et al. Interrupções e carga de trabalho de enfermagem durante a administração de medicamentos. Revista Brasileira de Enfermagem, 2019; 72(4): 1056-62.

20. SILVA ACA, et al. A segurança do paciente em âmbito hospitalar: revisão integrativa da literatura. Cogitare Enferm, 2016; 21(1): 01-09.

21. SILVA AT, et al. Patient safety and the nurse's performance in hospital. Rev enferm UFPE online. 2018; 12(6): 15321538.

22. SIMAN AG, et al. Practice challenges in patient safety. Rev Bras Enferm. 2019; 72(6): 1504-11.

23. SOUZA MJ, et al. Práticas Seguras Para Administração De Medicamentos: Construção E Validação De Instrumento. Enfermagem em Foco, 2018; 8(4): 20-25.

24. SLAWOMIRSKI L, et al. The economics of patient safety: strengthening a value-based approach to reducing patient harm at national level. Paris (France), OECD, 2017; 15(1): 63-70.

25. SCHWENDIMANN R, et al. The occurrence, types, consequences and preventability of in-hospital adverse events - a scoping review. BMC Health Serv Res, 2018; 18(521).

26. TELLES F, et al. Administração de medicamentos: aquisição de conhecimentos e habilidades requeridas por um grupo de enfermeiros. Revista Latino-americana de Enfermagem, 2004; 12(3): 533-540.

27. TEIXEIRA TCA, et al. Root cause analysis: evalutaion of medication errors at a university hospital. Rev Esc Enferm USP, 2010; 44(1): 139-46.

28. TOMASI YT, et al. Atuação do enfermeiro na administração de medicamentos em uma instituição hospitalar: estudo descritivo. Enferm Foco, 2015; 1(6): 6-11

29. VÓRIA JO, et al. Adesão às barreiras de segurança no processo de administração de medicamentos na pediatria. Texto Contexto Enferm, 2020; 29: e20180358.

30. WATSON G. The Hospital Safety Crisis. Society, 2016; 53(4): 339-47.

31. WORLD HEALTH ORGANIZATION (WHO). Medication without harm - Global Patient Safety Challenge. Geneva: WHO, 2017; 6-14. Disponível em: https://www.who.int/patientsafety/medication-safety/medication-without-harmbrochure/en/. Acessado em: 21 de março de 2021.

32. WORLD HEALTH ORGANIZATION (WHO). WHO global strategy on integrated people centred health services. 2015; 2016-2026. Disponível em: https://www.who.int/servicedeliverysafety/areas/people-centred-care/en/. Acessado em 22 de março de 2021.

33. WORLD HEALTH ORGANIZATION (WHO). The Conceptual Framework for the International classification. 2009; 32 41. Disponível em: https://www.who.int/patientsafety/taxonomy/icps_full_report.pdf/Acessado em 25 de março de 2021.

34. ZSIFKOVITS J, et al. Costs of unsafe care and cost-effectiveness of patient safety programmes. European Union: European Comission. Health and Food Safety, 2016; 28(1). 